\title{
Dexmedetomidine protects against renal ischemia and reperfusion injury by inhibiting the P38-MAPK/TXNIP signaling activation in streptozotocin induced diabetic rats ${ }^{1}$
}

\author{
Xiao Yeda', Lei Shaoqing", Huang Yayi"', Zhao Bo'v, Wang Huaxin'v, Cao HongV, Xia Zhongyuan
}

\begin{abstract}
'Master, Department of Anesthesiology, Renmin Hospital, Wuhan University, China. Conception and design of the study, acquisition and interpretation of data, manuscript writing.

"PhD, Department of Anesthesiology, Renmin Hospital, Wuhan University, China. Acquisition of data, critical revision.

I'PhD, Master, Department of Anesthesiology, Renmin Hospital, Wuhan University, China. Acquisition of data.

"Bachelor, Department of Anesthesiology, Wuhan the Third Hospital, China. Acquisition of data.

${ }^{v}$ Full Professor, Department of Anesthesiology, Renmin Hospital, Wuhan University, China. Design and supervised all phases of the study, critical revision.
\end{abstract}

\begin{abstract}
Purpose: To determine whether dexmedetomidine (DEX) could attenuate acute kidney injury (AKI) induced by ischemia/reperfusion (I/R) in streptozotocin (STZ)-induced diabetic rats.

Methods: Four groups each containing six rats were created (sham control(S), diabetes-sham (DS), diabetes I/R (DI/R), and diabetes-I/R-dexmedetomidine (DI/R-DEX). In diabetes groups, single-dose (65 mg/kg) STZ was administered intraperitoneally (i.p.). In Group DI/R, ischemia reperfusion was produced via 25 min of bilateral renal pedicle clamping followed by $48 \mathrm{~h}$ of reperfusion. In Group DI/R-DEX, $50 \mu \mathrm{g} / \mathrm{kg}$ dexmedetomidine was administered intraperitoneally 30 minutes before ischemia. Renal function, histology, apoptosis, the levels of TNF- $\alpha$, IL$1 \beta$, and oxidative stress in diabetic kidney were determined. Moreover, expression of P38 mitogen-activated protein kinase (P38-MAPK), phosphorylated-P38-MAPK(p-P38-MAPK) and thioredoxin-interacting protein (TXNIP) were assessed.

Results: The degree of renal I/R injury was significantly increased in DI/R group compared with $S$ group and DS group. The levels of TNF- $\alpha, I L-1 \beta$, oxidative stress and apoptosis were found significantly higher in DI/R Group when compared with S Group and DS Group. The protein expression of p-P38-MAPK and TXNIP were significantly increased after I/R. All these changes were reversed by DEX treatment.

Conclusion: The renoprotective effects of DEX-pretreatment which attenuates I/R-induced AKI were partly through inhibition of P38-MAPK activation and expression of TXINP in diabetic kidney.
\end{abstract}

Key words: Dexmedetomidine. p38 Mitogen-Activated Protein Kinases. Diabetes Mellitus. Kidney. Ischemia. Reperfusion. Rats. 


\section{- Introduction}

Acute kidney injury(AKI) is a common syndrome that is associated with incrased morbidity rates and affects many hospitalized patients1. The foremost causes of AKI include ischemia-reperfusion(l/R) injury, hypoxia or nephrotoxicity ${ }^{1}$, all of which is involved in excessive production of reactive oxygen species (ROS) to initiate a cascade of deleterious cellular responses ${ }^{2}$. Diabetes has been identified as an independent risk factor for $\mathrm{AKI}^{3}$, and $\mathrm{AKI}$ will be more serious in diabetic condition ${ }^{4-5}$. This is related to inflammatory response and apoptosis. However, the underlying mechanisms in which inflammatory reaction and oxidative stress exerts adverse effects in ischemia-reperfusion induced $\mathrm{AKI}$ are not clear.

The thioredoxin(TRX) as a ROS scavenging system keep the balance of intracelluar reduction/ oxidation ${ }^{6}$. TRX-interacting protein(TXNIP) is an intracelluar endogenous inhibitor of TRX'. It is reported that TXNIP could be up-regulated by high glucose in HK-2 cells ${ }^{8}$, and is involved in podocyte injury during diabetic nephropathy(DN) ${ }^{9}$. Moreover, TXNIP contributes to myocardial ischemia/reperfusion injury in diabetic mice ${ }^{10}$. However, the role of TXNIP in diabetic kidney to ischemia injury is unknown.

P38 mitogen-activated protein kinase (P38-MAPK) plays an important role in intracellular responses. Activation of $\mathrm{P} 38$ pathway is involved in human renal disease, including diabetic nephropathy and acute renal failure ${ }^{11}$. Previous studies have demonstrated that P38MAPK pathway contributes to the HG-induced upregulation of TXNIP expression and further increases intracellular ROS level ${ }^{12}$. However, the influence of P38-MAPK on TXNIP expression in combined ischemia and diabetes is unknown.

Dexmedetomidine (DEX), as a highly selective $\alpha 2$-adrenergic receptors agonist, is used for clinical anesthesia ${ }^{13}$. Pharmacologic benefits of DEX include sedation, analgesia, as well as anti-inflammatory ${ }^{14}$. Moreover, the beneficial effects of DEX have been showed to ameliorate I/R injury(l/RI) in many organs including kidney ${ }^{15}$ and recent studies have showed the protective effects of the brain and heart through anti-inflammatory and anti-apoptotic and anti-oxidative stress in diabetic rats ${ }^{16-17}$. However, the protective effects of DEX in diabetic kidney to ischemia-reperfusion injury have not been thoroughly investigated. Therefore, this study aims to investigate the protective effects of DEX in kidney against diabetic combined I/R induced injury. We hypothesized that DEX could suppress inflammatory response and oxidative stress in diabetic kidney during I/RI. Furthermore, we examined whether DEX could attenuate $\mathrm{I} / \mathrm{R}$ I in diabetic kidney through inhibiting P38-MAPK activation and TXNIP expression.

\section{- Methods}

\section{Animals grouping and induction of diabetes}

All the experiments complied with Ethics Committee of Renmin Hospital of Wuhan University. Twenty-four male Sprague-Dawley rats (250-300g, purchased from Beijing HFK Bioscience Co. Ltd, Beijing, China.) were used in this study. All the rats were maintained in the animal center of Wuhan University and kept under constant conditions at a temperature of $25 \pm 1^{\circ} \mathrm{C}$ with a $12 \mathrm{~h}$ dark/light cycle and had free access to food and water for one week before setting up the diabetic model.

Twenty-four rats were randomly divided into four groups each consisting of six rats: Group S(non-diabetic rats were used as sham control group); Group DS(diabetic rats were used as diabetic sham control group); Group DI/ $\mathrm{R}$ (diabetic rats were subjected to I/R); and Group DI/R-DEX(diabetic rats received $50 \mu \mathrm{g} / \mathrm{kg}$ of DEX (Hengrui, Jiangsu, China) injected intraperitoneally (i.p.) 30 minutes prior to $\left.I / R^{15}\right)$. Diabetes was induced by a single injection of STZ $(65 \mathrm{mg} / \mathrm{kg}$, i.p.) (Sigma-Aldrich, St Louis. MO, USA) which was 
dissolved in citrate buffer (0.1 Molar, $\mathrm{pH} 4.5)$. Two weeks after the STZ-induced injection, animals had a blood glucose level more than $300 \mathrm{mg} /$ dl were accepted as type-1diabetic ${ }^{18}$. These rats were used 4 weeks after STZ-induced5. Prior to the induction of $I / R$, diabetic rats were not given food but had free access for drinking.

\section{Establishment of rat kidney $I / R$ model}

Renal I/R was induced as previously described $^{5}$. Briefly, the rats were anesthetized through intraperitoneal injection pentobarbital sodium $(60 \mathrm{mg} / \mathrm{kg})$. The anesthetized rats were placed on a thermostatically controlled heating pad and kept body temperature at $37^{\circ} \mathrm{C}$. A middle laparotomy was performed. The kidneys were located inside the peritoneum and the renal pedicles were carefully isolated. The rats were subjected to bilateral renal occlusion for $25 \mathrm{~min}$ using nontraumatic artery clamps to clamp the renal pedicles, followed by reperfusion for 48 h. After the clamps were removed, the kidneys were observed for further $5 \mathrm{~min}$ to ensure reflow. Rats in the sham group underwent laparotomy without performing renal ischemia as controls. Rats were given 1-2 ml of warm saline intraperitoneally and kept in a warm incubator until they regained consciousness.

Blood sample were obtained from the left ventricle at the time point of $48 \mathrm{~h}$ after renal I/R, and centrifuged at $4000 \mathrm{rpm}$ for 15 min. Serum was stored at $-20^{\circ} \mathrm{C}$ for further biomedical determination. After $48 \mathrm{~h} \mathrm{I} / \mathrm{R}$, the right kidney was snap frozen at $-80^{\circ} \mathrm{C}$ and the left kidney was immediately post-fixed with $4 \%$ paraformaldehyde, dehydrated with ethanol and embedded in paraffin for further analysis.

\section{Histology and apoptosis examination}

\section{4\% paraformaldehyde-fixed renal tissues were dehydrated, embedded in paraffin and sliced into $4 \mu \mathrm{m}$ thick sections which were stained with hematoxylin and}

eosin. Histopathological evaluation under light microscopy was performed, the percentage of tubular injury as indicated by tubular epithelial swelling, loss of brush border, vacuolar degeneration, necrotic tubules, cast formation, and desquamation. The degree of kidney damage was estimated using five randomly selected fields for each rat assessed using quantitative analysis with the following criteria: $0=$ no abnormalities; $1=$ slight $(<25 \%$ involvement of the cortex or outer medulla); $2=$ moderate $(25-50 \%$ involvement of the cortex or outer medulla); $3=$ severe $(50-75 \%$ involvement of the cortex or outer medulla); and 4=more severe abnormalities (>75\% involvement of the cortex or outer medulla).

Renal apoptosis was examined by TUNEL assay using the in situ Apoptosis Detection kit from Roche Applied Science. TUNEL-positive cells were identified through the nucleus, which was either stained tan or brown. Five fields were randomly selected and the apoptosis index was calculated as the ratio of apoptotic-to-total cells.

All experimental analyzes were done by two examiners who were initially blinded to the experiment.

\section{Biochemical analysis}

Serum creatinine and plasma urea concentrations were measured by commercial kits (Jiancheng Biotech, Nanjing, China) to indicate renal function. The quantification of TNF- $\alpha$ and IL-1 $\beta$ was carried out by using the TNF- $\alpha$ and IL-1 $\beta$ commercial enzyme-linked immunosorbent assay (ELISA) kit following the manufacturer's instructions (Elabscience Biotechnology Co.,Ltd, Wuhan, China).

\section{Measurement of malondialdehyde (MDA), superoxide dismutase (SOD)}

The malondialdehyde (MDA) levels, superoxide dismutase(SOD) were detected 
using commercially-available kits(Jiancheng Biotech, Nanjing, China), according to manufactuer, instructions.

\section{Immunohistochemistry (IHC)}

The IHC staining for TXNIP expression in renal tissues was performed on formalinfixed, paraffin-embedded samples; $4 \mu \mathrm{M}$ sections were deparaffinized in graded xylenealcohol solutions. Subsequently, the samples were subjected to antigen retrieval and then incubated in $3 \% \mathrm{H}_{2} \mathrm{O}_{2} 15$ min then washed by PBS. The sections were incubated overnight (15 hours) at $4^{\circ} \mathrm{C}$ with a primary antibody anti-TXNIP (1:50) and then incubated with horseradish peroxidase-conjugated antiIgG secondary antibody for 20-30 min. The reaction was visualized with a solution of diaminobenzidine (DAB) and counterstained with hematoxylin.

\section{Western blot analysis}

The expressions of TXNIP, P38-MAPK and $\mathrm{p}$-P38-MAPK were measured using Western blot. Protein content was determined with BCA protein assay and protein samples were separated by electrophoresis on SDSPAGE and transferred to a polyvinylidene difluoride membrane. The membranes were blocked with $5 \%$ milk and incubated overnight with the appropriate primary antibodies respectively(Anti-TXNIP, 1:500, Abcam, USA. Anti-p38 and anti-p-p38, 1:1000, CST, USA), followed by incubation with the corresponding secondary antibodies. The blots were visualized with ECL-plus reagent. GAPDH was used as the internal loading control.

\section{Statistical analysis}

Data are expressed as the means \pm SE in each group. Experimental results were analyzed by one-way ANOVA followed by Tukey's test for multiple comparisons between the means of each group. All statistical analyses were performed using GraphPad Prism software version 5.0 (GraphPad Software, San Diego, CA, USA). A value of $\mathrm{P}<0.05$ was considered to indicate a statistically significant difference.

\section{- Results}

Body weight and blood glucose levels

The diabetic rats showed both increased blood glucose levels (Figure 1A) and reduced body weight (Figure 1B) significantly over 4 weeks. The results showed the success of the model of diabetes.
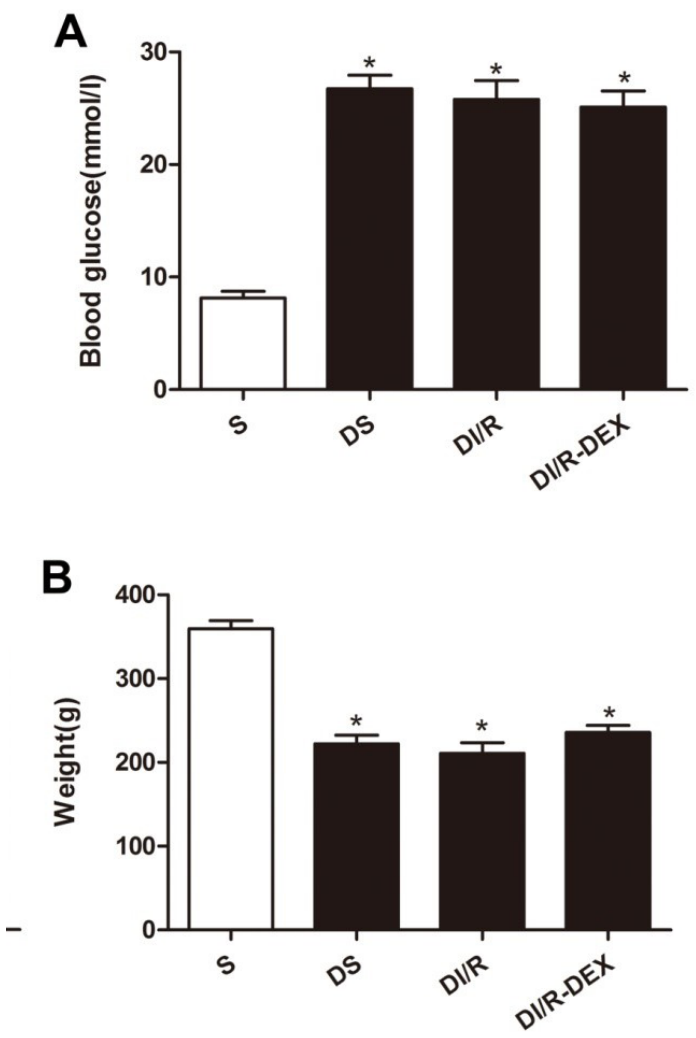

Figure 1 - Blood glucose (A) and body weight (B) were measured before surgery. Data are presented as the means $\pm S E(n=6) .{ }^{*} P<0.05$ vs $S$ group. 
DEX treatment attenuated histological lesion and improved renal function

In histopathological evaluation, we found normal morphology of tubular architecture and tubular cells in the $S$ group (Figure 2A), and mild damage was observed in the DS group (Figure $2 \mathrm{~B}$ ). In contrast, diabetic renal $I / R$ resulted in severe tubular damage in the DI/R group, the destruction included tubular epithelial swelling, loss of brush border, vacuolar degeneration, necrotic tubules, cast formation, and desquamation (Figure 2C). However, the injury was significantly ameliorated in the DI/R-DEX group (Figure 2D). The histopathological scores of renal tubular injury were presented (Figure 2E). The scores in the DI/R group were significantly higher than that in the DS group and also in the $S$ group. However, the injury was significantly attenuated in DI/R-DEX group when compared to the DI/R group. In the $\mathrm{DI} / \mathrm{R}$ group, serum creatinine and plasma urea (BUN) levels were significantly higher than those measured in $\mathrm{S}$ group and DS group, indicating renal dysfunction in the DI/R group. In the DI/R-DEX group, serum creatinine and BUN levels were significantly lower than those measured in DI/R group (Figure $2 \mathrm{~F}$ and $\mathrm{G}$ ).
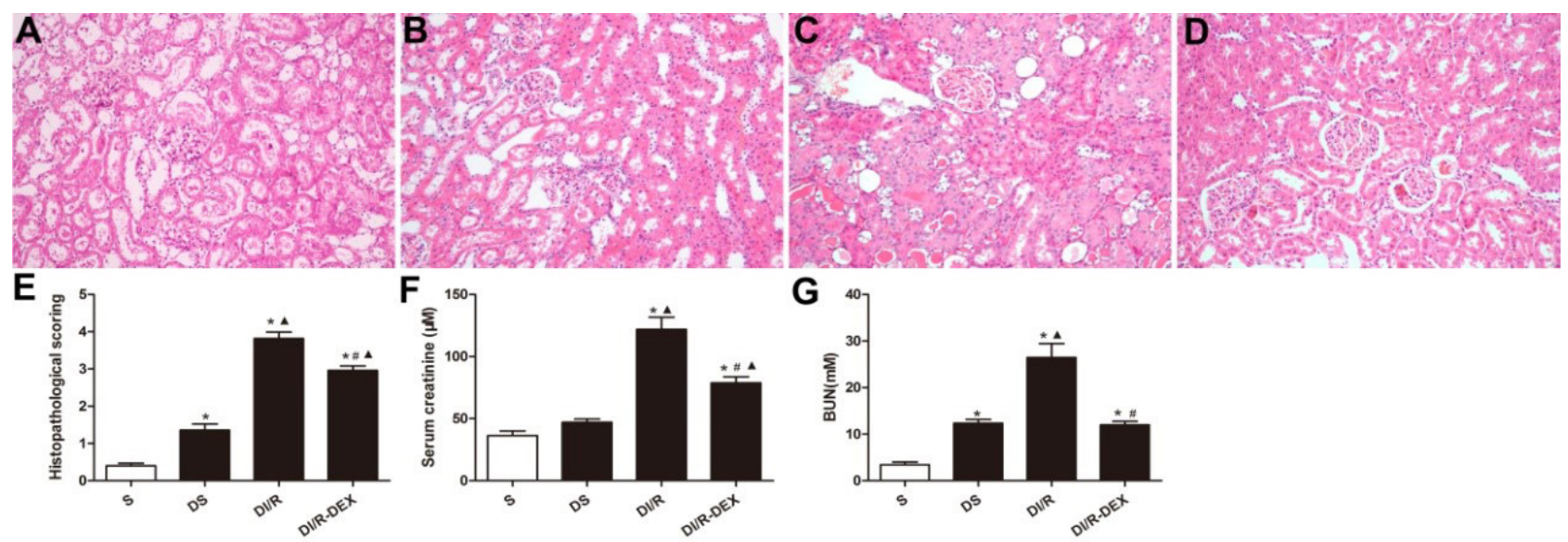

Figure 2 - Histological evaluation of renal tissue in four groups( $\times 400)$ : (A) S group; (B) DS group; (C) DI/R group; (D) DI/R-DEX group. Histopathological scoring (E). Serum creatinine (F) and BUN (G) were measured after $48 \mathrm{~h}$ reperfusion. Data are presented as the means $\pm S E(n=6)$., $* P<0.05$ vs $S$ group, $\triangle P<0.05$ vs $D S$ group. $\# \mathrm{P}<0.05$ vs DI/R group.

Effect of DEX on apoptosis of tubular epithelial cells

To evaluate the apoptosis of tubular epithelial cells induced by renal $\mathrm{I} / \mathrm{R}$ in diabetic rats, the TUNEL assay was used. A large number of apoptotic cells were visible in the DI/R group $(\mathrm{P}<0.05$ vs. the $\mathrm{S}$ group and the $\mathrm{DS}$ group). However, DEX treatment was associated with the occurrence of apoptosis ( $P<0.05$ vs. the $S$ group and the DS group) which was less than seen in the DI/R group (Figure 3). 

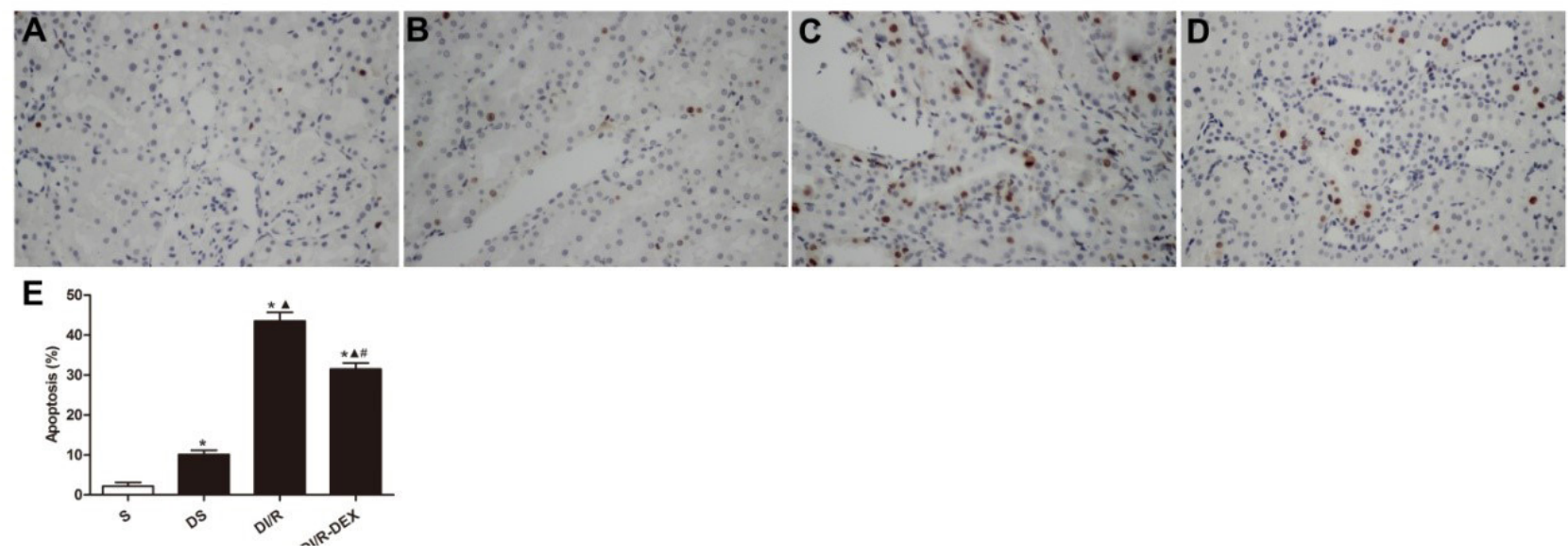

Figure 3 - Apoptosis of tubular epithelial cells of renal tissue in four groups ( $\times 400)$. (A) S group; (B) DS group; (C) DI/R group; (D) DI/R-DEX group. Apoptosis\% was assayed by TUNEL staining. Apoptosis \% (E). Data are presented as the means $\pm S E(n=6)$. ${ }^{*} P<0.05$ vs $S$ group, ${ }^{\triangle} P<0.05$ vs $D S$ group. $\# P<0.05$ vs $D I / R$ group.

Effect of DEX on inflammation and oxidative stress level

Inflammatory cytokines TNF- $\alpha$ (Figure $4 A$ ) and IL-1 $\beta$ (Figure 4B) were markedly higher in DI/R group than that in $S$ group and DS group. Treatment with DEX significantly decreased TNFaand IL-1ßlevels as compared with DI/R group.
Additionally, diabetic rats underwent renal $I / R$ exhibited a significant increase of MDA (Figure 4C) concentrations associated with significant reduction in SOD activity (Figure 4D) in comparison with the $\mathrm{S}$ group and DS group. The oxidative stress level associated with I/R was improved by the treatment with DEX in comparison with DI/R group.
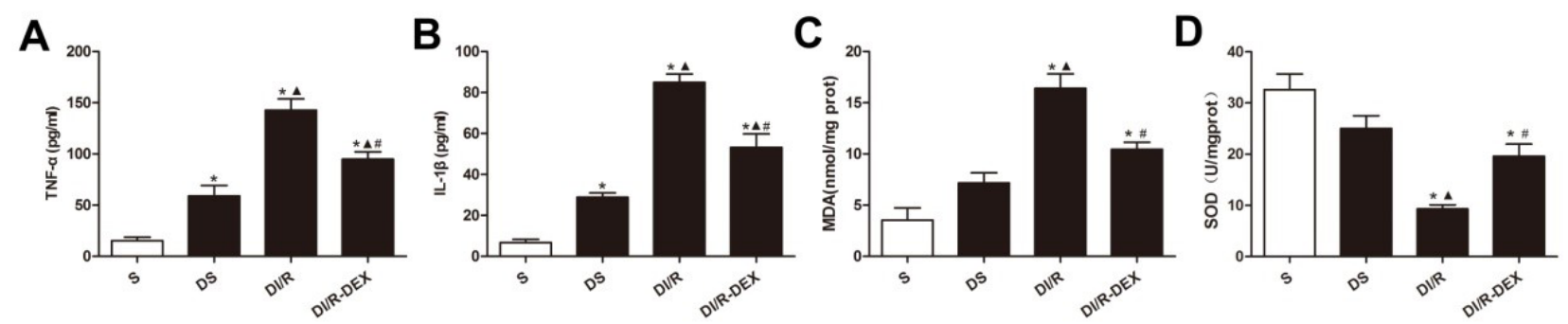

Figure 4 - TNF- $\alpha$ (A), IL-1B (B), MDA (C) and SOD (D) were measured after $48 \mathrm{~h}$ reperfusion. Data are presented as the means $\pm S E(n=6)$. ${ }^{*} P<0.05$ vs $S$ group, $\triangle P<0.05$ vs $D S$ group. $\# P<0.05$ vs $D I / R$ group.

Effect of DEX on the expression of p38-MAPK and TXNIP in I/R diabetic kidney

TXNIP protein was mainly expressed in renal tubular epithelial cells. DS group had week expression of TXNIP (Figure 5A). Immunohistochemical staining showed augmented expression of TXNIP protein in the $\mathrm{DI} / \mathrm{R}$ group (Figure 5B). The expression of TXNIP decreased in the DI/R-DEXgroup (Figure 5C). 

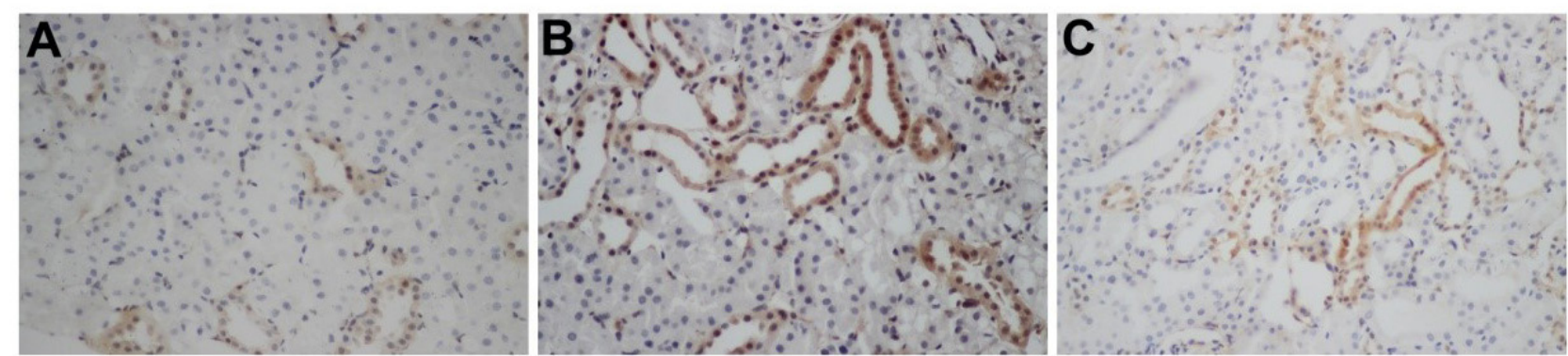

Figure 5 - Immunohistochemical staining of the TXNIP expression level (×400). (A) DS group; (B) DI/R group; (C) DI/R-DEX group.

We performed western blot to analyse the phosphorylation of P38-MAPK (p-P38) (Figure 6A-B) and expression of TXNIP (Figure $6 C)$. Western blotting revealed that p-P38 and TXNIP protein was low expression in the DS group. p-P38 and TXNIP protein expression
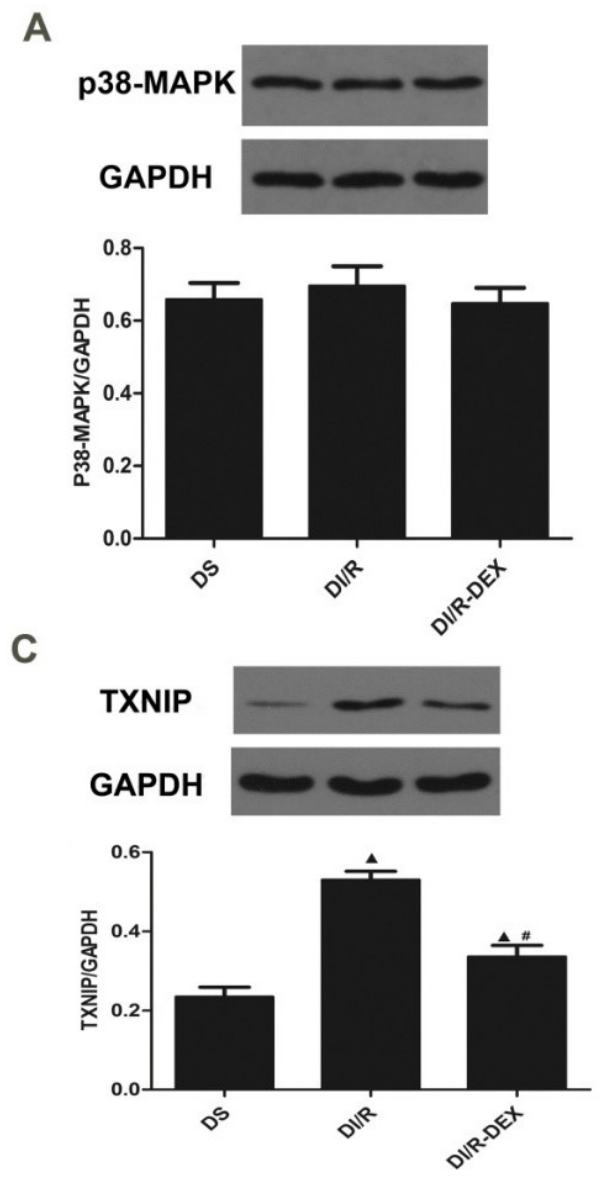

in the renal tissue in the $D I / R$ group was significantly higher than DS group. I/R induced p-P38 and TXNIP protein expression was significantly inhibited in DI/R-DEX group. The expression of P38 had no significantly change among three groups.
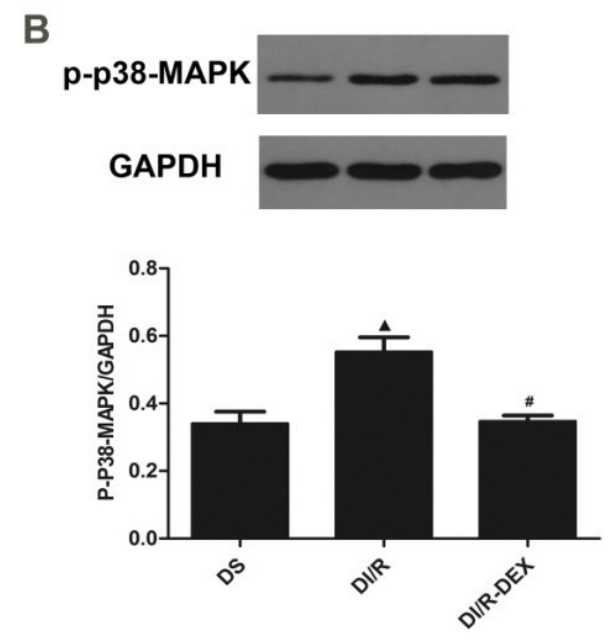

Figure 6 - Western blot analysis of p38 (A), p-P38 (B) and TXNIP (C) protein expression. Data are presented as the means $\pm S E(n=5) .{ }^{\triangle} P<0.05$ vs $D S$ group. $\# P<0.05$ vs $D I / R$ group. 


\section{- Discussion}

In this study, we demonstrated that DEX improved renal function, inhibited oxidative stress, inflammation and apoptosis in $\mathrm{I} / \mathrm{R}$ challenged diabetic kidney. The mechanism about this protection, at last in part, involved important role of the P38-MAPK/TXNIP signaling pathways.

It was well known that diabetic kidney was susceptibility to $\mathrm{I} / \mathrm{R}$ injury. However, the possible mechanisms and therapeutic strategies are still poorly understood. Previous researches demonstrated that may be related to oxidative stress $^{19}$, inflammation activition ${ }^{4}$ and apoptosis ${ }^{5}$. Effects of DEX with antioxidant and anti-inflammatory were shown in diabetic animals combined organs $I / R^{16-17,20}$. In our present study, which in accordance with previous study, renal I/R in diabetic rats induced high levels of apoptosis and oxidative stress (indicated by MDA and SOD), enhanced release of IL-1 $\beta$ and TNF- $\alpha$. Futhermore, treatment of DEX could significantly alleviate renal I/R injury in diabetic rats (indicated by histological scores, BUN and $\mathrm{SCr}$ ), suggesting a new strategy for renoprotection. However, the concrete mechanism remain further investigation.

TXNIP regulate the celluar redox state by binding to and inhibting TRX, is influenced by a variety of stress stimuli including hyperglycaemia ${ }^{8}$, hypoxia ${ }^{21}$ and so on. It was reported that increasing of TXNIP was major contributior to hyperglycaemia-induced oxidative stress in HK-2 cells ${ }^{8}$ and mediated IL-1 $\beta$ transcription in Human Adipose Tissue ${ }^{22}$. IL-1 $\beta$ is an important mediator of inflamation in $\mathrm{I} / \mathrm{R}$ injury ${ }^{23}$. Recently study showed that lessening TXNIP expression in diabetic mice could protects against myocardial ischemia/ reperfusion injury ${ }^{10}$. However, the role of TXNIP about I/R injury in diabetic kidney have not been elucidated. In this research, for the first time to our knowledge, showed that I/R induced TXNIP expression up-regulation in diabetic kidneys. Moreover, DEX pretreatment suppressed
TXNIP expression and reduced oxidative stress and IL-1 $\beta$ production. Thus we speculate that TXNIP may be a potential therapeutic target for ischemia AKI in diabetic kidney.

P38-MAP kinase, one of three subgroups of MAPK systems, is involved in the regulation of cellular function. Different stimuli such as inflammatory, oxidative stress, and hyperglycemia could activate P38-MAPK ${ }^{24}$. Recent study showed that a novel mechanism for P38-MAPK cause kidney I/R injury by leading redox stress and cell apoptosis, suggested it is a potential therapeutic target of tissue injury ${ }^{25}$. Meldrum, K.K further demonstrated that P38-MAPK mediates renal tubular cell TNFaproduction and TNF- $\alpha$-dependent apoptosis during simulated ischemia ${ }^{26}$. However, there is no study examined the P38-MAPK pathway in combining ischemia-reperfusion and diabetes. Datas from our study also indicated that I/R insulted diabetic kidney significantly increased phosphorylation of P38-MAPK, accompanied with high levels of TNF-aand apoptosis. More importantly, TNF- $\alpha$ as a key inflammatory factor which involved in increasing susceptibility with ischemia AKI of $\mathrm{db} / \mathrm{db}$ mice $^{4}$. Our result further demonstrated that suppressed P38MAPK pathway by DEX in diabetic kidney could not only markedly reduced P38-MAPK phosphorylation, but also decreased TXNIP expression and oxidative stress level. Other studies had also reported that upregulation of TXNIP impaired TRX antioxidative system through P38-MAPK and FOXO1 for glucoseinduced increase in intracellular $\operatorname{ROS}^{12}$. Su et al found that hyperglycemia promoted the TXNIP expression and results in the exacerbation of cardiac injury following $\mathrm{I} / \mathrm{R}$ possibly through P38-MAPK activation ${ }^{27}$. Furthermore, hyperglycemia-induced TXNIP expression is involved in diabetes-mediated oxidative stress in pancreatic cancer via P38 MAPK pathways ${ }^{28}$. These observations indicated that P38-MAPK as an upstream mechanism for TXNIP expression during hyperglycemia even hyperglycemia combined with I/R-induced injury. 
DEX had been shown to improve renal dysfunction and inflammation in renal ischemic injury ${ }^{15}$. Moreover, DEX attenuated myocardial I/R induced lung damage in STZinduced diabetic rats through the modulation of oxidative stress ${ }^{20}$. Previous study reported that DEX protected against brain and heart ischemia injury in diabetic rats by reducing oxidative stress and inflammation ${ }^{16-17}$. Other research indicated that DEX-mediated decrease of the JNK and P38-MAPK pathways which inhibited isoflurane-induced neuroapoptosis ${ }^{29}$. Meanwhile, DEX attenuates isoflurane-induced cognitive impairment through antioxidant, antiinflammatory and anti-apoptosis in aging rat by inhibition of P38-MAPK expression ${ }^{30}$.Our present research has shown that induced oxidative and inflammation-mediated diabetic kidney tubular cell apoptosis is ameliorated by DEX via preventing P38-MAPK phosphorylation and TXNIP expression. Thus, DEX may be a candidate for development of a multipotent drug.

Taken together, our data provide a molecular basis that treatment with DEX favourably inhibited the P38-MAPK/ TXNIP pathways which further prevented inflammation, oxidative stress and apoptosis in the I/R induced renal injury in diabetic rats.

\section{Conclusions}

Our work provide a new molecular basis (p38-MAPK/TXNIP pathway) for DEX to be a potential therapeutic for AKI in diabetic patients.

\section{References}

1. Basile DP, Anderson MD, Sutton TA. Pathophysiology of acute kidney injury. Compr Physiol. 2012;2(2):1303-53. doi: 10.1002/cphy.c110041.

2. Malek M, Nematbakhsh M. Renal ischemia/ reperfusion injury; from pathophysiology to treatment. J Renal Inj Prev. 2015;4(2):20-7. doi: 10.12861/jrip.2015.06.

3. Venot $M$, Weis L, Clec'H C, Darmon M, Allaouchiche B, Goldgran-Toledano D,
Garrouste-Orgeas M, Adrie C, Timsit JF, Azoulay $\mathrm{E}$. Acute kidney injury in severe sepsis and septic shock in patients with and without Diabetes mellitus: a multicenter study. PLoS One. 2015;10(5):e127411. doi: 10.1371/journal.pone.0127411.

4. Gao G, Zhang B, Ramesh G, Betterly D, Tadagavadi RK, Wang W, Reeves WB. TNFalpha mediates increased susceptibility to ischemic AKI in diabetes. Am J Physiol Renal Physiol. 2013 Mar 1;304(5):F515-21. doi: 10.1152/ajprenal.00533.2012.

5. Peng J, Li X, Zhang D, Chen JK, Su Y, Smith SB, Dong Z. Hyperglycemia, p53, and mitochondrial pathway of apoptosis are involved in the susceptibility of diabetic models to ischemic acute kidney injury. Kidney Int. 2015;87(1):137-50. doi: 10.1038/ ki.2014.226.

6. Ray PD, Huang BW, Tsuji Y. Reactive oxygen species (ROS) homeostasis and redox regulation in cellular signaling. Cell Signal. 2012;24(5):981-90. doi: 10.1016/j. cellsig.2012.01.008.

7. Spindel ON, World C, Berk BC. Thioredoxin interacting protein: redox dependent and independent regulatory mechanisms. Antioxid Redox Signal. 2012;16(6):587-96. doi: 10.1089/ars.2011.4137.

8. Wei J, Shi Y, Hou Y, Ren Y, Du C, Zhang L, Li $Y$, Duan HDuan. Knockdown of thioredoxininteracting protein ameliorates high glucose-induced epithelial to mesenchymal transition in renal tubular epithelial cells. Cell Signal. 2013;25(12):2788-96. doi: 10.1016/j. cellsig.2013.09.009.

9. Gao P, Meng XF, Su H, He FF, Chen S, Tang $H$, Tian XJ, Fan D, Wang YM, Liu JS, Zhu ZH, Zhang $C$. Thioredoxin-interacting protein mediates NALP3 inflammasome activation in podocytes during diabetic nephropathy, Biochim Biophys Acta. 2014;1843(11):244860. doi: 10.1016/j.bbamcr.2014.07.001.

10.Zhu HJ, Wang DG, Yan J, Xu J. Up-regulation of microRNA-135a protects against myocardial ischemia/reperfusion injury by decreasing TXNIP expression in diabetic mice. Am J Transl Res. 2015;7(12):2661-71. PMID: 26885264. 
11.Ma FY, Liu J, Nikolic-Paterson DJ. The role of stress-activated protein kinase signaling in renal pathophysiology. Braz J Med Biol Res. 2009;42(1):29-37. doi: 10.1590/00$879 \times 2008005000049$.

12.Li X, Rong $\mathrm{Y}$, Zhang $\mathrm{M}$, Wang $\mathrm{XL}$, LeMaire SA, Coselli JS, Zhang Y, Shen YH. Up-regulation of thioredoxin interacting protein (Txnip) by p38 MAPK and FOXO1 contributes to the impaired thioredoxin activity and increased ROS in glucose-treated endothelial cells. Biochem Biophys Res Commun. 2009;381(4):660-5. doi: 10.1016/j.bbrc.2009.02.132.

13.Niu XY, Ding XB, Guo T, Chen MH, Fu SK, Li Q. Effects of intravenous and intrathecal dexmedetomidine in spinal anesthesia: a meta-analysis. CNS Neurosci Ther. 2013;19(11):897-904. doi: 10.1111/ cns.12172.

14.Sanders RD, Maze M. Alpha2-adrenoceptor agonists. Curr Opin Investig Drugs. 2007;8(1):25-33. PMID: 17263182.

15.Si Y, Bao H, Han L, Shi H, Zhang Y, Xu L, Liu C, Wang J, Yang X, Vohra A, Ma D. Dexmedetomidine protects against renal ischemia and reperfusion injury by inhibiting the JAK/STAT signaling activation. J Transl Med. 2013;11:141. doi: 10.1186/1479-587611-141.

16.Zeng X, Wang $H$, Xing X, Wang Q, Li W. Dexmedetomidine protects against transient global cerebral ischemia/reperfusion induced oxidative stress and inflammation in diabetic rats. PLoS One. 2016;11(3): e151620. doi: 10.1371/journal.pone.0151620.

17.Arslan M, Comu FM, Kip G, Alkan M, Kiraz HA, OzerA, Sivgin V. Effect of dexmedetomidine on erythrocyte deformability during ischaemiareperfusion injury of heart in diabetic rats. Bratisl Lek Listy. 2014;115(8):494-7. PMID: 25246286.

18.Chang CC, Chang CY, Wu YT, Huang JP, Yen TH, Hung LM. Resveratrol retards progression of diabetic nephropathy through modulations of oxidative stress, proinflammatory cytokines, and AMP-activated protein kinase. J Biomed Sci. 2011;18(1):47. doi: 10.1186/1423-0127-18-47.

19.Abu-Saleh N, Awad H, Khamaisi M, Armaly Z,
Karram T, Heyman SN, Kaballa A, Ichimura T, Holman J, Abassi Z. Nephroprotective effects of TVP1022, a non-MAO inhibitor S-isomer of rasagiline, in an experimental model of diabetic renal ischemic injury. Am J Physiol Renal Physiol. 2014;306(1): F24-33. doi: 10.1152/ajprenal.00379.2013.

20.Kip G, Çelik A, Bilge M, Alkan M, Ali Kiraz $H$, Özer A, Şıvgın V, Erdem Ö, Arslan $M$, Kavutçu M. Dexmedetomidine protects from post-myocardial ischaemia reperfusion lung damage in diabetic rats. Libyan J Med. 2015;10:27828. doi: 10.3402/ljm.v10.27828.

21.Baker AF, Koh MY, Williams RR, James B, Wang H, Tate WR, Gallegos A, Von Hoff DD, Han $\mathrm{H}$, Powis G. Identification of thioredoxininteracting protein 1 as a hypoxia-inducible factor 1alpha-induced gene in pancreatic cancer. Pancreas. 2008;36(2):178-86. doi: 10.1097/MPA.0b013e31815929fe.

22.Koenen TB, Stienstra R, van Tits $L J$, de Graaf J, Stalenhoef AF, Joosten LA, Tack CJ, Netea MG. Hyperglycemia activates caspase-1 and TXNIP-mediated IL-1beta transcription in human adipose tissue. Diabetes. 2011;60(2):517-24. doi: 10.2337/db100266.

23.Wanderer AA. Ischemic-reperfusion syndromes: biochemical and immunologic rationale for IL-1 targeted therapy. Clin Immunol. 2008;128(2):127-32. doi: 10.1016/j.clim.2008.03.514.

24.Kyriakis JM, Avruch J. Mammalian mitogenactivated protein kinase signal transduction pathways activated by stress and inflammation. Physiol Rev. 2001;81(2):80769. PMID: 11274345.

25.Ashraf MI, Ebner M, Wallner C, Haller $M$, Khalid S, Schwelberger $H$, Koziel $K$, Enthammer $M$, Hermann $M$, Sickinger $S$, Soleiman A, Steger C, Vallant S, Sucher R, Brandacher G, Santer P, Dragun D, Troppmair J. A p38MAPK/MK2 signaling pathway leading to redox stress, cell death and ischemia/reperfusion injury. Cell Commun Signal. 2014;12:6. doi: 10.1186/1478-811X12-6.

26. Meldrum KK, Meldrum DR, Hile KL, Yerkes EB, Ayala A, Cain MP, Rink RC, Casale AJ, 
Kaefer MA. p38 MAPK mediates renal tubular cell TNF-alpha production and TNF-alpha-dependent apoptosis during simulated ischemia. Am J Physiol Cell Physiol. 2001;281(2):C563-70. PMID: 11443055.

27.Su H, Ji L, Xing W, Zhang W, Zhou H, Qian $X$, Wang X, Gao F, Sun X, Zhang H. Acute hyperglycaemia enhances oxidative stress and aggravates myocardial ischaemia/ reperfusion injury: role of thioredoxininteracting protein. J Cell Mol Med. 2013;17(1):181-91. doi: 10.1111/j.15824934.2012.01661.x.

28.Li W, Wu Z, Ma Q, Liu J, Xu Q, Han L, Duan W, Lv Y, Wang F, Reindl KM, Wu E. Hyperglycemia regulates TXNIP/TRX/ROS axis via p38 MAPK and ERK pathways in pancreatic cancer. Curr Cancer Drug Targets. 2014;14(4): 348-56. PMID: 24720336.

29.Liao Z, Cao D, Han X, Liu C, Peng J, Zuo Z, Wang F, Li Y. Both JNK and P38 MAPK pathways participate in the protection by dexmedetomidine against isofluraneinduced neuroapoptosis in the hippocampus of neonatal rats. Brain Res Bull. 2014;107:6978. doi: 10.1016/j.brainresbull.2014.07.001. 30.Wang X, Zhao B, Li X. Dexmedetomidine attenuates isoflurane-induced cognitive impairment through antioxidant, antiinflammatory and anti-apoptosis in aging rat. Int J Clin Exp Med. 2015;8(10):17281-8. PMID: 26770320.

\section{Correspondence:}

Xia Zhongyuan

Department of Anesthesiology, Renmin Hospital, Wuhan University

Jiefang Road 238

Wuhan, Hubei, China, 430060

Phone: (86)027-88041911(82024)

Xiazhongyuan2005@aliyun.com

\section{Conflict of interest: none}

Financial source: The Basic Scientific Research of Central University Fund (2042017kf0144)
Received: Feb 04, 2017

Review: Apr 06, 2017

Accepted: May 08, 2017
${ }^{1}$ Research performed at Central Laboratory, Renmin Hospital, Wuhan University, Hubei, China. 